\title{
Investigating the psychometric properties of the Jefferson Scale of Physician Empathy in a sample of Malaysian medical students
}

This article was published in the following Dove Press journal:

Advances in Medical Education and Practice

6 June 2016

Number of times this article has been viewed

\author{
Marija Spasenoska' \\ Shane Costello' \\ Brett Williams ${ }^{2}$ \\ 'Faculty of Education \\ ${ }^{2}$ Department of Community \\ Emergency Health and Paramedic \\ Practice, Monash University, \\ Melbourne, VIC, Australia
}

Correspondence: Brett Williams Department of Community Emergency Health and Paramedic Practice, Monash University - Peninsula Campus, PO Box 527, McMahons Road, Frankston, Melbourne, VIC 3199, Australia

Tel +6I 399044283

Fax +6I 399044168

Email brett.williams@monash.edu
Objective: The purpose of this present study was to investigate the psychometric properties of the Jefferson Scale of Physician Empathy - student version (JSPE-S).

Subjects and methods: This study recruited 193 Malaysian medical students enrolled in year one and year two studies. A principal-component analysis with Varimax rotation was conducted. Procrustes rotation was used to confirm the item to model fit, which allows for a comparison of actual structure against an ideal hypothesized structure. Items were systematically removed based on low communalities of $<0.3$ and poor loading of items onto components.

Results: A two-component solution was found, comprised of "perspective taking" and "compassionate care". Following item removal, eleven items remained. A Procrustes analysis revealed that this eleven-item measure demonstrated an excellent model fit. A possible third component was identified, though is not recommended for use, due to construct underrepresentation.

Conclusion: This study found the Jefferson Scale of Physician Empathy fitted best to a twocomponent model using eleven items. Item, component, and overall congruence were very high, and scale reliabilities were adequate. The results of this study suggest that the eleven-item, two-component solution demonstrates excellent psychometric properties and structural validity in a Malaysian medical student population. Future research could consider using the short eleven-item measure in both student and health care profession samples to investigate the role of empathy in health care.

Keywords: empathy, medical students, psychometrics

\section{Background}

The concept of empathy in medical settings is crucial, as it contributes to positive patient-care outcomes. ${ }^{1,2}$ It is thus important to utilize psychometrically validated measures in order to measure empathy in health care workers, such as physicians and nurses. Empathy can often become a widely misunderstood and devalued concept in society, as its importance in health care settings is often overlooked. In order to overcome this misunderstanding, the concept of empathy needs to be further reiterated in order to make it widely known that empathy is multidimensional in nature, involving core concepts underlying its definition., ${ }^{2,3}$ Empathy is recognized to be influential upon positive patient-care outcomes, although the concept of empathy is often mistakenly confused with the concept of sympathy. ${ }^{2-4}$ It appears clear that further distinctions need to be made between the concepts of sympathy and empathy, as although they can be interrelated, they are two separate and unique constructs. ${ }^{2-4}$ Recent work by Cuff et al in their reconceptualization of empathy provided an important context for ongoing research on empathy in health care. ${ }^{5}$ They suggested that there are functional differences 
in empathy and interrelated constructs, particularly between cognition and emotions, and that this distinction can have an impact on patient outcomes and medical education. ${ }^{5}$

This present study aims to investigate the psychometric properties of the Jefferson Scale of Physician Empathy student version (JSPE-S), a 20-item empathy measure, when applied to a sample of Malaysian medical students.

\section{What is empathy?}

Empathy in relation to patient care allows for an understanding of an individual's inner experience while maintaining a sense of emotional detachment, as emotions can work to cloud one's judgment, which can thus interfere with maintaining objectivity. ${ }^{1,3}$ Hojat viewed empathy as having two core components - cognitive and affective - which are attached to its meaning. ${ }^{2}$ Empathy relies on higher-order cognition, as opposed to sympathy, as there is an increased level of cognitive information processing involved when one must reflect on another's situation. ${ }^{1}$ This involves imagining what it would be like for that person and taking on their perspective, in order to experience what it would be like to be in their situation. ${ }^{1}$ Wispé defined empathy as the ability to comprehend one's experiences nonjudgmentally. ${ }^{4}$

\section{Empathy in patient-care situations}

Empathy in the medical arena has become increasingly important, as it enhances patient satisfaction and wellbeing. ${ }^{1,2}$ With its links to "prosocial" and helping behavior, empathy has been associated with altruism, which is the act of helping another purely out of concern, without the intention or expectation of being rewarded for that helping behavior. ${ }^{2}$ The process of understanding in empathy allows for physician metaphorically to put themselves in the patient's shoes, allowing the observer to gain insight into another person's thoughts, feelings, and behavior. ${ }^{1}$ Empathy in patient-care settings allows the physician to develop an understanding into the patient's experiences and for the formation of a therapeutic relationship between the physician and patient. ${ }^{6}$ Empathy involves active components of sharing and conveying the physician's understanding back to the patient, all while maintaining a sense of emotional detachment. ${ }^{3}$ Overall, empathy in patient care has been viewed positively, as it has been known to increase patient satisfaction, decrease levels of stress in patients, and increase compliance with treatment regimens..$^{6-8}$

Past research has suggested that sympathy and empathy are often confused. ${ }^{2-4}$ It therefore needs to be further recognized that even though they are interrelated concepts, empa- thy and sympathy are two differing and unique concepts. ${ }^{3,9}$ Physician empathy contributes to positive patient-care outcomes, so it is important that it is not confused with sympathy.

Sympathy in patient care is seen as more of an emotional reaction to the patient's distress, rather than adopting an understanding, which distinguishes empathy from sympathy. ${ }^{3}$ In sympathy, emotions tend to be heightened, which interferes with one's ability to remain objective. Wispé viewed emotions as being distorted in sympathy, so one can lose this ability to remain objective. ${ }^{4}$ This is different to empathy, as the higher-order cognition involved in empathy allows for one to maintain objectivity in a situation. ${ }^{2}$

General measures of empathy include the Interpersonal Reactivity Index, ${ }^{10}$ the Empathy Scale, ${ }^{11}$ the Consultation and Relational Empathy measure, ${ }^{12}$ and the Emotional Empathy Scale. ${ }^{13}$ These general measures of empathy were not specifically designed to measure empathy among physicians in their delivery of health care services. This has prompted the development of the Jefferson Scale of Physician Empathy (JSPE), which is one of the most widely used and psychometrically tested measure of empathy. ${ }^{14}$

\section{The Jefferson Scale of Physician Empathy}

The importance of physician empathy in patient care has prompted the development of the JSPE. The JSPE was developed by an extensive review of the literature, wherein factor analysis was employed for the refinement and development of a 20-item empathy measure. ${ }^{14}$ The JSPE is a 20-item empathy measure that is designed to assess empathy in physicians on a 7-point Likert scale. ${ }^{14}$ The JSPE has also been adapted for use in medical students, nurses, and health care professional students, labeled, respectively, as the JSPE-S and the health care provider version (JSPE-HP). ${ }^{2}$ A concern with the JSPE is the variation of factor structures that have been reported in the literature: either two or three factors emerge, which suggests that further research on structural (or construct) validity is warranted.

Numerous studies have applied the JSPE within varying populations, such as in physicians, nurses, health care practitioners, and students. For example, Hojat et al administered the JSPE to a sample of 704 physicians using a principalcomponent analysis (PCA) with orthogonal rotation. ${ }^{15}$ The authors found a three-factor solution: "perspective taking", with ten items loading, "compassionate care", with eight items loading, and "standing in the patient's shoes", with only two items loading. ${ }^{15}$ Retaining these three factors was based on using Kaiser's criteria, which state that any eigenvalues exceeding the value of 1 should be retained. ${ }^{16}$ This approach 
has been criticized, as it can result in retaining too many factors. ${ }^{17,18}$

Similarly, Hsiao et al administered the Chinese version of the JSPE-HPS to a sample of 613 undergraduate nursing students. ${ }^{19}$ This study used an exploratory factor analysis with Oblimin rotation, where three factors were retained - "perspective taking", with ten items loading, "compassionate care", with eight items loading, and "standing in the patient's shoes", again with only two items loading which was consistent with Hojat et al. ${ }^{15}$ The decision on how many factors to retain was based on Kaiser's criteria. ${ }^{16}$ Similar findings emerged in Suh et al, who administered the Korean-translated version of the JSPE-HP to a sample of 229 physicians, where three factors also emerged. ${ }^{20} \mathrm{~A}$ PCA with Varimax rotation was used in order to investigate the underlying structure of the JSPE. The authors also used Kaiser's criteria and the scree plot, where one retains the eigenvalues above the bend or break in the curve, to assist in their decision in determining how many factors to retain. ${ }^{16,17,21}$ The scree-plot test has been criticized in the past, as its interpretation is subjective, so it is difficult to provide evidence for its accuracy. ${ }^{18}$

Williams et al used PCA in order to investigate the psychometric properties of the JSPE-HPS when applied to a sample of 330 paramedic students. ${ }^{8}$ To assist in their decision on how many factors to retain, the authors used Kaiser's criteria, where eigenvalues $>1.25$ were used and retained, inspection of the scree test, cumulative percentage of variance extracted, and a parallel analysis. ${ }^{8,16,21}$ This study found a two-factor solution, with nine items loading onto factor one labeled "compassionate care" and eight items loading onto factor two - "perspective taking".

Factor analysis on the JSPE and its variations presents results with a two- and sometimes three-factor structure, with components including "perspective taking", "compassionate care", and occasionally "standing in the patient's shoes". This lack of consistency is of concern to researchers, given the challenge to validity posed by attempting to draw meaningful comparisons between studies that have utilized different measures.

Due to the inconsistencies present in the current literature concerning the factor structure of the JSPE, and the fact that no previous study has examined this using a Malaysian medical student cohort, the aim of this current study was thus to assist in confirming the factorial structure of the JSPE. The current study sought to investigate the psychometric properties and underlying component structure of the JSPE-S when applied to a sample of Malaysian medical students. Previous findings support a two- or three-factor structure of the JSPE; however, the three-factor structures reported has been found to demonstrate insufficient or inconsistent factor loadings. At present, no studies have sought to confirm the structure of the JSPE using targeted rotation to an ideal matrix; therefore, the current study investigated the component structure of the JSPE using a Procrustes analysis. Due to previous studies rejecting a third factor present in the JSPE due to insufficient items loading on the third factor, this study hypothesized that two factors would emerge - "perspective taking" and "compassionate care" - while the third factor, "standing in the patient's shoes", would be underrepresented. Undertaking such a study is important in developing robust and culturally appropriate measurement tools relating to empathy.

\section{Subjects and methods Design}

SPSS was used to conduct the PCA and analyze the data. The study design used PCA with Varimax rotation, analyzing all variance, followed by a Procrustes confirmatory analysis. The values generated from the PCA by the Kaiser-Meyer-Olkin (KMO) test and Bartlett's test of sphericity were used to determine the suitability of the data for PCA. A Procrustes rotation allows the component solution to be compared to an ideal matrix, in which the items load completely or not at all. ${ }^{22}$ The aim of a Procrustes rotation is to provide information about the overall fit of the items on the model..$^{22}$ In research, when conducting a PCA, which yields components, as opposed to conducting a factor analysis, which yields factors, with both techniques being similar, the terms "factor" and "component" are often used interchangeably. It is therefore reasonable to assume that if the author lists the term "factor", they may be using PCA. ${ }^{18,23}$ A Monte Carlo parallel analysis was conducted, which generates a random data set to which eigenvalues are compared. ${ }^{24}$

\section{Instrumentation}

The JSPE is a 20 -item self-report questionnaire that measures a respondent's level of empathy on a 7-point Likert scale ranging from 1 (strongly disagree) to 7 (strongly agree). Scores on the JSPE-S can range from 20 to 140, indicating that the higher one scores on the JSPE, the more empathetic they are said to be. ${ }^{14}$ The JSPE-S version includes ten positively worded items labeled under "perspective taking". In the JSPE-S version, ten items were negatively worded, including 
six "compassionate care" items, two "standing in the patient's shoes" items, and two items that did not load onto any factor. These negatively worded items were reverse scored on a Likert scale of 7 (strongly disagree) to 1 (strongly agree). ${ }^{25}$ In this study, the JSPE-S was not translated into another language, as all students spoke English and had completed their school education in English.

\section{Participants}

The participants for this current study were 193 students enrolled in years one and two studying medicine at the Jeffry Cheah School of Medicine and Health Sciences, Monash University, Sunway, Malaysia. The students were from different ethnic backgrounds, including Chinese, Indian, Malaysian, and "other", as shown in Table 1. From the 193 students who participated in this study, 19 were identified as international students. The mean age of participants was 19.3 years, with a standard deviation of 1.24 years. All students in this study spoke English, as they were taught using the English language and had completed their schooling in English.

\section{Ethics}

Approval to undertake this study was obtained from Monash University Human Ethics Committee. All procedures contributing to this work complied with the ethical standards of the relevant national and institutional committees on human experimentation and with the Helsinki Declaration of 1975 , as revised in 2008 .

Table I Participant demographics ( $\mathrm{N}=$ 193)

\begin{tabular}{lllllll}
\hline $\begin{array}{l}\text { Demographic } \\
\text { variable }\end{array}$ & \multicolumn{2}{l}{ Combined } & \multicolumn{2}{l}{ Male } & \multicolumn{3}{l}{ Female } \\
\cline { 2 - 7 } Nex & $\%$ & $\mathbf{N}$ & $\%$ & $\mathbf{N}$ & $\%$ \\
\hline Male & 83 & 43 & - & - & - & - \\
$\quad$ Female & 110 & 57 & - & - & - & - \\
Nationality & & & & & & \\
$\quad$ Chinese & 121 & 62.7 & 58 & 69.9 & 63 & 57.3 \\
Indian & 41 & 21.2 & 14 & 16.9 & 27 & 24.5 \\
Malaysian & 10 & 5.2 & 5.0 & 6.0 & 5.0 & 4.5 \\
$\quad$ Other & 21 & 10.9 & 6.0 & 7.2 & 15 & 13.6 \\
Age, years & & & & & & \\
$\quad$ Minimum & 17 & - & 17 & - & 17 & - \\
$\quad$ Maximum & 30 & - & 23 & - & 30 & - \\
$\quad$ Mean & 19.8 & - & 19.7 & - & 19.9 & - \\
$\quad$ SD & 1.2 & - & 1.1 & - & 1.4 & - \\
Year of study & & & & & & \\
Ist year & 122 & 63.2 & 54 & 65.1 & 68 & 61.8 \\
2nd year & 71 & 36.2 & 29 & 34.9 & 42 & 38.2 \\
\hline Ab
\end{tabular}

Abbreviation: SD, standard deviation.

\section{Procedure}

First- and second-year students enrolled in medicine were invited to participate in the research during their orientation day and at the end of a lecture. Recruitment into the study was undertaken by a nonteaching staff member. Students who opted into the study completed the survey in the lecture theater and returned completed surveys into a cardboard box near the exit. The JSPE-S survey was paper based, and participation in the study was voluntary. Responses were anonymous, and consent was implied through the completion and return of the survey.

\section{Statistical analysis}

SPSS version 20 and Orthosim version 2.01 were used to conduct the statistical analysis. Initial screening of the data indicated no missing values, and normality was assessed, in which no violations were found. Outliers were screened for and found not to be an issue. Skewness and kurtosis values did not exceed 1, which supported the data's suitability for analysis. ${ }^{23}$ Suitability of the data was determined using the KMO measure of sampling adequacy (should be 0.6 or greater) and Bartlett's test of sphericity (should be significant, ie, $P<0.05)$. PCA with Varimax rotation was conducted in order to determine the underlying dimensions of the JSPE-S, which was followed by a targeted rotation to an ideal matrix, where items are specified a priori to load either perfectly or not at all to individual factors and measures of how well those items fit are calculated. The decision to remove items was based on whether the commonalities displayed a value of $<0.3$ and also how weakly the items loaded onto a component. Loadings above the recommended 0.32 were interpreted. ${ }^{23}$

\section{Results}

An initial PCA was conducted, which revealed a seven-component solution with eigenvalues $>1$ accounting for $59.55 \%$ of the cumulative variance. The KMO measure was 0.77 , and Bartlett's test was significant at $\chi^{2}=343.124, d f=55$, and $P \leq 0.001$, supporting the factorability of the data. Inspection of the scree plot, however, showed a clear bend after the second component. Following this, a Monte Carlo parallel analysis revealed that three eigenvalues should be retained when compared against a random data set. ${ }^{24}$

The three-component solution was extracted as shown in Table 2, with the first component explaining $19.42 \%$ of variance, the second component explaining $9.5 \%$ of the variance, and the third component explaining $7.45 \%$ of the variance.

Inspection of the three-component solution, however, revealed only three items loading on it, although there was 
a good item-to-component fit. Given the limited number of items and the lack of cohesion among the items following a visual inspection of the item content, a two-component solution was suggested.

A two-component solution was forced, and the total variances extracted were $27.13 \%$ and $9.76 \%$ for the two components. Upon inspection of the two-component solution, several poorly fitting items were identified and systematically removed based on poor communalities of $<0.30$, low primary loadings, and significant secondary loadings. This was done until a simple structure was achieved, with all items demonstrating a primary loading $>0.32$ and minimal secondary loading. ${ }^{26}$ Adequate reliability was demonstrated for the two extracted factors, with the first factor displaying Cronbach's $a=0.74$ with an interitem correlation range of $0.168-0.397$, and for the second factor Cronbach's $\alpha=0.60$ with an interitem correlation range of $0.119-0.384$ ), with most values exceeding $0.30 .{ }^{23}$ As shown in Table 3, component one was labeled "perspective taking" and component two was labeled "compassionate care".

Once a two-component solution was established, the data were transformed using a Procrustes rotation using Orthosim version 2.1. ${ }^{27}$ The result of the Procrustes rotation is also presented in Table 3. The application of a Procrustes analysis to the data revealed a near-perfect item-to-model fit, with items demonstrating very high congruence with the a priori "perspective taking" and "compassionate care" constructs. At the component level, the items for "perspec- tive taking" demonstrated a congruence of 0.99 , while the items for "compassionate care" demonstrated a congruence of 0.97 . The overall solution congruence (or model fit) was 0.98 , with scores $>0.95$ indicating excellent congruence.

\section{Discussion}

This study investigated the psychometric properties of the JSPE-S in a sample of Malaysian medical students. Results from this study supported our hypothesis of a two-component solution. Our PCA with orthogonal rotation revealed the presence of a two-component solution, with the first component labeled "perspective taking" and the second component "compassionate care". This reflects the core components highlighted within the definition of empathy presented in this paper, with "perspective taking" reflecting the cognitive components and "compassionate care" reflecting the affective components. The two-component solution found in our study varies from other non-Malaysian psychometric papers in the literature; one potential reason for this could be that Malaysians are traditionally very tolerant and altruistic, and have an upbringing of respecting rights of others. ${ }^{28}$ Further qualitative research is required to understand these structural differences fully.

Similar results were produced in the study of Fjortoft et al on pharmacy students, which also used a PCA with orthogonal rotation, revealing a two-component solution consisting of "perspective taking" and "compassionate care". ${ }^{29}$ These two components are described as core aspects

Table 2 Initial three-component rotated matrix $(n=193)$

\begin{tabular}{|c|c|c|c|}
\hline Item & Factor I & Factor 2 & Factor 3 \\
\hline 20. I believe that empathy is an important therapeutic factor in medical treatment & 0.70 & 0.06 & 0.01 \\
\hline 9. Physicians should try to stand in their patients' shoes when providing care for them & 0.67 & -0.18 & -0.14 \\
\hline 4. Understanding body language is as important as verbal communication in the physician-patient relationship & 0.64 & -0.09 & 0.00 \\
\hline 10. Patients value a physician's understanding of their feelings, which is therapeutic in its own right & 0.62 & -0.16 & -0.14 \\
\hline 2. Patients feel better when their physicians understand their feelings & 0.60 & 0.01 & -0.03 \\
\hline 16. Physicians' understanding of the emotional status of patients, as well as that of their families, is important & 0.58 & 0.25 & 0.34 \\
\hline 13. Physicians should understand what is going on by paying attention to nonverbal cues and body language & 0.55 & -0.15 & 0.05 \\
\hline I5. Empathy is a therapeutic skill without which the physician's success is limited & 0.53 & -0.02 & 0.01 \\
\hline 17. Physicians should try to think like their patients in order to render better care & 0.48 & -0.20 & 0.10 \\
\hline 5. A physician's sense of humor contributes to a better clinical outcome & 0.37 & -0.00 & 0.13 \\
\hline 8. Attentiveness to patients' experiences does not influence treatment outcomes & -0.04 & 0.66 & 0.21 \\
\hline II. Patients' illnesses can be cured by medical or surgical treatment & -0.12 & 0.64 & 0.04 \\
\hline 12. Asking patients about what is happening in their personal lives is not helpful & -0.03 & 0.63 & -0.15 \\
\hline I. Physicians' understanding of their patients' feelings does not influence medical or surgical treatment & -0.06 & 0.56 & -0.01 \\
\hline 14. I believe that emotion has no place in the treatment of medical illness & -0.18 & 0.49 & 0.12 \\
\hline 7. Attention to patients' emotions is not important in history taking & -0.12 & 0.38 & 0.04 \\
\hline 19. I do not enjoy reading nonmedical literature or the arts & 0.04 & 0.31 & -0.27 \\
\hline 6. Because people are different, it is difficult to see things from patients' perspectives & 0.06 & 0.07 & 0.79 \\
\hline 3. It is difficult for a physician to view things from patients' perspectives & 0.04 & 0.07 & 0.69 \\
\hline 18. Physicians should not allow themselves to be influenced by strong personal bonds with patients & 0.01 & 0.03 & 0.36 \\
\hline
\end{tabular}

Notes: Bolded loadings signify item allocation. Item descriptions have been reworded for space. Factor I is labeled "perspective taking", factor 2 "compassionate care", and factor 3 "standing in the patient's shoes". 
Table 3 Final two-component rotated matrix and Procrustes rotation ( $\mathrm{N}=193)$

\begin{tabular}{|c|c|c|c|c|c|c|}
\hline Item & Comm & Factor I & Factor 2 & PL & PL & CC \\
\hline 9. Physicians should try and stand in their patients when providing care & 0.53 & 0.71 & -0.17 & 0.98 & -0.21 & 0.98 \\
\hline 2. Patients feel better when their physicians understand their feelings & 0.49 & 0.70 & 0.04 & 1.00 & -0.08 & 1.00 \\
\hline 16. Physicians' understanding of the emotional status of patients is important & 0.47 & 0.65 & -0.22 & 0.95 & -0.31 & 0.95 \\
\hline 20. I believe that empathy is an important therapeutic factor in medical treatment & 0.42 & 0.65 & 0.01 & 1.00 & 0.04 & 1.00 \\
\hline 4. Understanding body language is as important as verbal communication & 0.42 & 0.64 & -0.07 & 1.00 & -0.08 & 1.00 \\
\hline 17. Physicians should try to think like their patients in order to render better care & 0.37 & 0.58 & -0.18 & 0.96 & 0.28 & 0.96 \\
\hline II. Patients' illnesses can be cured only by medical/surgical treatment & 0.51 & -0.05 & 0.71 & -0.09 & 1.00 & 1.00 \\
\hline 8. Attentiveness to patients' experiences does not influence treatment outcomes & 0.48 & -0.04 & 0.69 & -0.07 & 1.00 & 1.00 \\
\hline 12. Asking patients about what is happening in their personal lives is not helpful & 0.40 & -0.04 & 0.63 & -0.09 & 1.00 & 1.00 \\
\hline 14. I believe that emotion has no place in the treatment of medical illness & 0.30 & -0.12 & 0.53 & -0.24 & 0.97 & 0.97 \\
\hline I. Physicians' understanding of their patients feelings and family does not influence treatment & 0.27 & -0.13 & 0.51 & -0.27 & 0.96 & 0.96 \\
\hline Factor-congruence values & & & & 0.99 & 0.98 & \\
\hline
\end{tabular}

Notes: Bolded loadings signify item allocation. Item descriptions have been reworded for space. Factor I is labeled "perspective taking" and factor 2 "compassionate care". Abbreviations: Comm, commonality; PL, Procrustes loading; CC, congruence coefficient.

of empathy, as "perspective taking" relates to the concept of understanding, which is encompassed within the definition of empathy, ${ }^{1,3}$ and the word "understanding" is also present in three of our six items loading on component one. Further, component two, "compassionate care", is concerned with the feelings and emotions associated with adopting an empathetic understanding. ${ }^{6,29}$

Initially, our analysis extracted seven components, although according to the scree plot, ${ }^{21}$ there appeared to be a clear break after the third point, with the parallel analysis confirming this. ${ }^{24}$ When the solution was fixed to three components, the rotated component matrix appeared to show only three stable items with primary loadings on the third component, which was labeled "standing in the patient's shoes", with item 18 ("Physicians should not allow themselves to be influenced by strong personal bonds between their patients and their family members"), item 3 ("It is difficult for a physician to view things from patients' perspectives"), and item 6 ("Because people are different, it is difficult to see things from patients' perspectives") loading strongly onto component three. However, as there were only three items loading, component three could not be labeled a stable component, as according to Costello and Osborne, a factor with three or fewer items is weak, as a stable factor should have at least five items that load onto it. ${ }^{17}$ Further, there appeared to be semantic concerns regarding the third component, as items 3 and 6 appeared very similar in content, with both displaying high loadings on the third component. This was different to the third item loading onto component three, item 18 , which differed considerably in meaning compared to items 3 and 6. Item 18 also displayed quite low loading on the third component, thus indicating that the third component displayed only two good items and one poor item.
This further influenced the decision to force the solution to two components. Items 3 and 6 both strongly loading onto component three is consistent with the studies of Kožený and Tišanská, ${ }^{30}$ Vallabh, ${ }^{25}$ and Ward et al, ${ }^{6}$ which all showed items 3 and 6 to load strongly onto factor three. Following this as the solution was forced into two components, it was found that both items 18 and 19 were deleted from the analysis, as they did not appear to load on either component one or two. Similar findings were presented in Vallabh's study, where neither item 18 nor 19 loaded onto any factor. ${ }^{25}$ Consistent findings were produced in Ward et al, ${ }^{6}$ who found that item 19 did not load onto any one of the three factors that emerged, and Williams et al, ${ }^{8}$ who found item 18 not to load onto any of the two factors that emerged.

Following this, inspection of the items was conducted, and the items that presented with the lowest commonalities and loaded the most weakly onto the components were deleted. This caused the deletion of item 5 - "A physician's sense of humor contributes to a better clinical outcome" - which loaded the most weakly onto component one - perspective taking - as a physician's sense of humor had no similarity to the other items that did load onto the perspective taking component, such as items relating to adopting a sense of understanding.

Both items 3 ("It is difficult for a physician to view things from patients' perspectives") and 6 ("Because people are different, it is difficult to see things from patients' perspectives"), which were "Standing in the patient's shoes" items, were deleted, as they were the only two "Standing in the patient's shoes" items that loaded onto the second component of "compassionate care" and presented with the lowest commonalities. With items 3 and 6, the scree plot and parallel analysis supported a three-component solution, as 
these items originally loaded onto the third component. ${ }^{21,24}$ Due to this, the third component was underrepresented, with only two items loading on it. Items 3 and 6 , which loaded highly onto component three, were tested and found to be reliable together. This suggests that the JSPE could in fact be a true three-component measure with further development of the JSPE-S scale, although the results from this study reveal that the JSPE-S produces only two stable components instead.

Item 15 ("Empathy is a therapeutic skill without which the physician's success is limited") was deleted, as this item's wording seemed to create confusion and thus could have been the reason that it loaded poorly on both components one (perspective taking) and two (compassionate care). Item 7 ("Attention to patients' emotions is not important in history-taking") was deleted, as it appeared to be too broad and not specific enough to be related to empathy, as it could be in regard to history taking for simply measuring blood pressure. Item 10 ("Patients value a physician's understanding of their feelings, which is therapeutic in its own right") was deleted, as it appeared as an item that originally did not fit in with either the "perspective taking" or "compassionate care" items, the decision to delete this item was also based on the confusion surrounding the item's wording.

Although item 10 was deleted in the current study and was an item that did not originally load onto any of the primary two components, it was considered to be a "perspective taking" item, as it contained the word "understanding", which is included as part of the wording in the other "perspective taking" items. In numerous studies, item 10 was revealed to be a "perspective taking" item, such as in Fjortoft et al, ${ }^{29}$ Williams et al, ${ }^{8}$ Ward et al, ${ }^{6}$ Hojat et al, ${ }^{15}$ Kožený and Tišanská, ${ }^{30}$ and Hsiao et al, ${ }^{19}$ although item 10 did not seem to load onto any of the three factors listed in Vallabh. ${ }^{25}$

Item 13 ("Physicians should try to understand what is going on in their patients' minds by paying attention to their nonverbal cues and body language"), a "perspective taking" item, was also deleted, as this item appeared to be loading the most lowly on component one in comparison to the other "perspective taking" items.

Item 4 ("Understanding body language is as important as verbal communication in the physician-patient relationship"), originally a "compassionate care" item, was also considered for deletion. However, item 4 was not deleted as it seemed to be presenting quite a high loading on component one: "perspective taking". When item 4 was inspected, it related to "perspective taking" more than to "compassionate care", as the concept of "understanding" consistently pre- sented itself among the wording in the "perspective taking" items, which further influenced the decision not to delete item 4. In numerous studies, item 4 loaded on "perspective taking", instead of "compassionate care", which provided us with further support to retain item 4 as a "perspective taking" item. . $, 8,15,19,29,30$

Implications of this study include the fact that the elevenitem, two-component solution demonstrates structural validity in a Malaysian medical student population, supporting its use for investigating empathy in this population. The modified eleven-item JSPE was demonstrated to be a good fit using Procrustes analysis.

\section{Limitations and future research}

The largest limitation for the current study involved the use of self-report. Given that the JSPE is a self-report measure, participants have the potential to answer in a socially desirable manner, thus not accurately reflecting their true intentions. Investigating medical professional empathy from the perspective of the patient would assist in addressing this limitation. Another potential limitation may have been the contextual nature of the questionnaire, particularly with firstand second-year medical students, who had experienced little to no clinical patient-care contact.

Suggestions for future research include further development of the underrepresented construct "Standing in the patient's shoes". This would involve developing additional items related to this construct, as the current study revealed only two items -3 and 6 - loaded strongly onto the third component. With only two items loading onto the third component, construct underrepresentation is a threat to the structural integrity of the instrument, given that the presence of two items is not enough to retain it as a separate component. ${ }^{15}$ This has previously been found in numerous studies, such as Kožený and Tišanská, ${ }^{30}$ Vallabh, ${ }^{25}$ and Ward et al, ${ }^{6}$ which adds support for further development of a third component of the JSPE, although the third component cannot be recommended at this time. Future research could also consider permanent deletion of items 18 and 19, as there have been inconsistencies surrounding their loadings on any of the three factors, which was replicated in the current study. These two items have been found not to load onto any component or factor in numerous studies, providing evidence for their permanent deletion.

\section{Conclusion}

This study found that the eleven-item JSPE-S formed a robust two-component structure, consisting of "perspec- 
tive taking" and "compassionate care" subscales. These subscales are consistent with previous research, while a third subscale - "Standing in the patient's shoes" - suffered from construct underrepresentation. For this construct to be able to demonstrate adequate psychometric properties, further item development is required. Empathy researchers are encouraged to consider using the eleven-item JSPE-S scale to assess empathy in medical students, as it is a reliable and well-fitting model. Using a reliable and shorter scale of eleven items to measure empathy is beneficial, as it results in shorter completion time for the individual. The results of the current study suggest that the use of the three-component structural model of the JSPE is not warranted and that the two-component model demonstrates considerably improved psychometric properties in the shorter eleven-item version.

\section{Acknowledgment}

The authors would like to thank the students who completed the surveys.

\section{Author contributions}

All authors contributed to study conception, collated and analyzed data, and helped write the paper. All authors read and approved the final manuscript.

\section{Disclosure}

The authors report no conflicts of interest in this work.

\section{References}

1. Coplan A, Goldie P. Empathy: Philosophical and Psychological Perspectives. Oxford: Oxford University Press; 2011.

2. Hojat M. Empathy in Patient Care: Antecedents, Development, Measurement, and Outcomes. Heidelberg: Springer; 2007.

3. Clark AJ. Empathy and sympathy: therapeutic distinctions in counseling. J Ment Health Couns. 2010;32(2):95-101.

4. Wispé L. The distinction between sympathy and empathy: to call forth a concept, a word is needed. J Pers Soc Psychol. 1986;50(2):314-321.

5. Cuff B, Brown S, Taylor L, Howat D. Empathy: a review of the concept. Emot Rev. Epub 2014 Dec 1.

6. Ward J, Schaal M, Sullivan J, Bowen ME, Erdmann JB, Hojat M. Reliability and validity of the Jefferson Scale of Empathy in undergraduate nursing students. J Nurs Meas. 2009;17(1):73-88.

7. Brown T, Boyle M, Williams B, et al. Predictors of empathy in health science students. J Allied Health. 2011;40(3):143-149.

8. Williams B, Brown T, Boyle M, Dousek S. Psychometric testing of the Jefferson Scale of Empathy Health Profession Students' version with Australian paramedic students. Nurs Health Sci. 2013;15(1):45-50.
9. Irving P, Dickson D. Empathy: towards a conceptual framework for health professionals. Int J Health Care Qual Assur Inc Leadersh Health Serv. 2004;17(4-5):212-220.

10. Davis MH. Measuring individual differences in empathy: evidence for a multidimensional approach. J Pers Soc Psychol. 1983;44(1):113-126.

11. Hogan R. Development of an empathy scale. J Consult Clin Psychol. 1969;33(3):307-316.

12. Mercera S, Maxwell M, Heaney D, Watta G. The consultation and relational empathy (CARE) measure: development and preliminary validation and reliability of an empathy-based consultation process measure. Fam Pract. 2004;21(6):699-705.

13. Mehrabian A, Epstein N. A measure of emotional empathy. $J$ Pers. 1972;40(4):525-543.

14. Hojat M, Mangione S, Nasca TJ, et al. The Jefferson Scale of Physician Empathy: development and preliminary psychometric data. Educ Psychol Meas. 2001;61(2):349-365.

15. Hojat M, Gonnella JS, Nasca TJ, Mangione S, Vergare M, Magee M. Physician empathy: definition, components, measurement, and relationship to gender and specialty. Am J Psychiatry. 2002;159(9):1563-1569.

16. Kaiser HF. The application of electronic computers to factor analysis. Educ Psychol Meas. 1960;20(1):141-151.

17. Costello AB, Osborne JW. Best practices in exploratory factor analysis: four recommendations for getting the most from your analysis. Pract Assess Res Eval. 2005;10(7):1-9.

18. Pallant J. SPSS Survival Manual: A Step by Step Guide to Data Analysis Using IBM SPSS. Maidenhead, UK: McGraw-Hill; 2013.

19. Hsiao CY, Tsai YF, Kao YC. Psychometric properties of a Chinese version of the Jefferson Scale of Empathy - health profession students. J Psychiatr Ment Health Nurs. 2013;20(10):866-873.

20. Suh DH, Hong JS, Lee DH, Gonnella JS, Hojat M. The Jefferson Scale of Physician Empathy: a preliminary psychometric study and group comparisons in Korean physicians. Med Teach. 2012; 34(6):e464-e468.

21. Cattell RB. The scree test for the number of factors. Multivariate Behav Res. 1966;1(2):245-276.

22. Jacobs KE, Costello S. An initial investigation of an Australian adaptation of the Multidimensional Aptitude Battery - II. Aust Educ Dev Psychol. 2013;30(1):84-102.

23. Tabachnick BG, Fidell LS. Using Multivariate Statistics. Boston: Pearson; 2014.

24. Watkins MW. Monte Carlo PCA for Parallel Analysis [software]. State College (PA): Ed \& Psych Associates; 2000.

25. Vallabh K. Psychometrics of the student version of the Jefferson Scale of Physician Empathy (JSPE-S) in final-year medical students in Johannesburg in 2008. S Afr J Bioeth Law. 2011;4(2):63-68.

26. Thurstone LL. Multiple Factor Analysis. Chicago: University of Chicago Press; 1947.

27. Barrett P. Orthosim 2 v.1: online help in manual form. 2006. Available from: http://imaging.mrc-cbu.cam.ac.uk/statswiki/FAQ/congruenceC?a ction=AttachFile \&do=get\&target=congruence.pdf. Accessed March 3, 2016.

28. Williams B, Sadasivan S, Kadirvelu A, Olaussen A. Empathy levels among first year Malaysian medical students: an observational study. Adv Med Educ Pract. 2014;16(5):149-156.

29. Fjortoft N, Van Winkle LJ, Hojat M. Measuring empathy in pharmacy students. Am J Pharm Educ. 2011;75(6):109.

30. Kožený J, Tišanská L. The structure of the Jefferson Scale of Physician Empathy in Czech physicians. Cesk Psychol. 2013;57(6):521. 
Advances in Medical Education and Practice

Dovepress

\section{Publish your work in this journal}

Advances in Medical Education and Practice is an international, peerreviewed, open access journal that aims to present and publish research on Medical Education covering medical, dental, nursing and allied health care professional education. The journal covers undergraduate education, postgraduate training and continuing medical education including emerging trends and innovative models linking education, research, and health care services. The manuscript management system is completely online and includes a very quick and fair peer-review system. Visit http://www.dovepress.com/testimonials.php to read real quotes from published authors.

Submit your manuscript here: http://www.dovepress.com/advances-in-medical-education-and-practice-journal 